\title{
Caracterización de la producción de miel de abeja en el departamento de Neembucú
}

\author{
Autor: Rolando David Acosta Bobadilla \\ rolyccp69@gmail.com \\ Tutora: MSc. Fulvia Karina Gamarra \\ Facultad de Ciencias Aplicadas \\ Universidad Nacional de Pilar \\ Pilar - Paraguay
}

\section{RESUMEN}

La apicultura se constituye en uno de los rubros más difundidos últimamente en el departamento de Ñeembucú. Esta actividad puede usarse como fuente de diversificación productiva, por lo que se hace necesario conocer la materia prima, el manejo que se está realizando en el proceso de producción y el impacto económico en las familias productoras, atendiendo que la apicultura constituye una importante actividad económica con un atractivo potencial convirtiéndose en alternativa de diversificación agropecuaria. El trabajo de investigación se titula "Caracterización de la producción de miel de abeja en el departamento de Ñeembucú", cuyo objetivo centra su interés en la producción de miel en tres zonas de mayor relevancia productiva. El trabajo se encuadra dentro de la modalidad de artículo científico. Corresponde a un tipo de estudio descriptivo, utilizando el método cualitativo- cuantitativo y de corte transversal. Como técnica de recolección de datos se implementará la entrevista, el análisis documental y el cuestionario. Los resultados permitirán principalmente caracterizar la producción en las zonas de influencia productiva y establecer planes de acción para el desarrollo apícola en el Departamento de Ñeembucú.

Palabras claves: producción; apicultura; miel de abeja 


\title{
Characterization of the production of honey in the department of Ñeembucú
}

\begin{abstract}
Beekeeping has become one of the most popular sectors lately in the department of Neembucú. This activity can be used as a source of productive diversification, so it is necessary to know the raw material, the management that is being carried out in the production process and the economic impact on the producer families, taking into account that beekeeping constitutes an important economic activity. with an attractive potential, becoming an alternative for agricultural diversification. The research work is titled "Characterization of the production of honey from bee in the department of Neembucú", whose objective focuses its interest in the production of honey in three areas of greater productive relevance. The work is framed within the modality of scientific article. It corresponds to a descriptive type of study, using the qualitative-quantitative and crosssectional method. As a data collection technique, the interview, the documentary analysis and the questionnaire will be implemented. The results will mainly allow characterizing the production in the areas of productive influence and establishing action plans for beekeeping development in the Department of Neembucú.
\end{abstract}

Keywords: production; beekeeping; honey

Artículo recibido: 02 enero 2022 Aceptado para publicación: 28 enero 2022

Correspondencia: rolyccp69@gmail.com Conflictos de Interés: Ninguna que declarar 


\section{INTRODUCCIÓN}

El Departamento de Ñeembucú se caracteriza por ser eminentemente agropecuario, los pequeños productores se hallan distribuidos entre grandes extensiones de campos de pastoreos, que la mayor parte se encuentran inundados. (Gamarra, 2008)

La vegetación natural predominante, presentan condiciones privilegiadas para la explotación racional de las abejas y los productos obtenidos son considerados de excelente calidad. Además, las perspectivas de mercado para los productos apícolas, se cree, son alentadoras, tanto en el mercado nacional e internacional.

Se considera además que Ñeembucú posee condiciones favorables para la explotación racional de las abejas melíferas, hallándose algún lugar libre aún de contaminantes de residuos tóxicos provenientes de la utilización de agrotóxico, factor fundamental para producir un producto orgánico.

Dadas las circunstancias y la experiencia que se tiene en el financiamiento de los implementos apícolas para los productores, apoyados por la asistencia técnica de manejo y capacitación periódica, es necesario el estudio de viabilidad de generación de producto orgánico ( Miel ) como uno de los rubros de renta y mejoramiento del nivel alimenticio de la familia rural y de la población urbana del Departamento y a través de ello la factibilidad de instalar un centro de acopio y envasado de miel de abeja.

La apicultura es un rubro que hoy en día nace como una posibilidad de desarrollo económico en el departamento y como un complemento en la diversificación de los rubros, elemento clave para el desarrollo sustentable en el área rural.

Dentro de este contexto la producción y comercialización adecuada de la miel de abeja, contribuirá al desarrollo apícola de la región, dando grandes posibilidades para que el hombre del campo obtenga mayores ingresos y mejore su calidad de vida.

Si bien existe pequeños comités y otros en proceso de formación que actúan en forma aislada, esta investigación nos llevará a encontrar propuestas viables para el desarrollo apícola en el departamento de Neembucú de manera a consolidar los comités de apicultores, a través de un Centro de Acopio y procesadora de productos apícolas, que nos permita la centralización de la producción / comercialización, y así abastecer el mercado interno de la ciudad de Pilar y la población de alrededores, el mercado nacional y proyectarse eventualmente a la exportación.- 


\section{Planteamiento del problema}

Con base a lo anterior, puede apreciarse, que, la miel es una sustancia dulce elaborada por las abejas a partir del néctar de las flores, las cuales recogen, combinan con sustancias específicas, transforman y almacenan en panales para servir posteriormente como alimento energético. La transformación de néctar a miel se produce debido a cambios físicos y químicos. Los primeros se deben principalmente a un proceso de evaporación, en el cual, el néctar pierde hasta una tercera parte de su contenido de humedad durante su almacenamiento en la colmena, y los segundos se deben a la acción de enzimas que las obreras adicionan al néctar, como es la invertasa (sacarasa), la cual hidroliza la sacarosa presente en el néctar a glucosa y fructose. ( Moguel Ordóńeza Y, Echazarreta Gonzalez C, Mora Escobedo R, 2005)

La apicultura se constituye en uno de los rubros más difundidos últimamente en el departamento de Neembucú por el MAG (Ministerio de Agricultura y Ganadería) a través del DEAG (Dirección de Extensión Agrícola y Ganadera) La apicultura se constituye en uno de los rubros más difundidos últimamente en el departamento de Neembucú por el MAG (Ministerio de Agricultura y Ganadería) a través del DEAG (Dirección de Extensión Agrícola y Ganadera). (Gamarra, 2008)

La composición química de la miel depende principalmente de las fuentes vegetales de las cuales se deriva, pero también de la influencia de factores externos, como el clima, el manejo de extracción y almacenamiento. Un mal manejo de la miel puede reducir su calidad; los factores que más influyen en ello son las altas temperaturas, el tiempo de almacenamiento y contenido de humedad superior a $21 \%$, los cuales ocasionan fermentaciones, formación de hidroximetilfurfural, pérdida de la actividad enzimática, cambio del sabor, obscurecimiento y crecimiento microbiano en la miel. ( Moguel Ordóńeza Y, Echazarreta Gonzalez C, Mora Escobedo R, 2005)

Según las estadísticas y estudios realizados, el departamento de Ñeembucú, es el departamento con mayor producción anual del país. Atendiendo este hecho, es importante obtener una buena calidad de la miel producida. Las condiciones de la calidad de la miel de abeja, están reguladas por la Comisión Codex- Normas Internacionales de los Alimentos, y establecida en el código Codex Stan 12-1981. Esta norma hace referencia a factores como ser la composición, su nivel de contaminación y su higiene. (Benítez, 2017) 
Dentro de esta investigación, lo que se pretende es centrar la caracterización de la miel dentro de los criterios de calidad que hacen a la composición, bajo tres aspectos fundamentales que son la cantidad de humedad, azucares y cantidad de sólidos insolubles En este caso, se analiza un grupo de comités, con determinados rasgos y condiciones enmarcados previamente como universo de estudio en una determinada población, que estarán conformadas por apicultores de las localidades de Gral. Díaz, Paso de Patria y Humaitá, técnicos especializados y entidades comercializadoras.

\section{El Departamento de Ñeembucú. Descripciones generales}

Ñeembucú se halla situado al sur del país, en el vértice formado por los ríos Paraná y Paraguay, entre los paralelos $25^{\circ} 45^{\prime}$ y $27^{\circ} 15^{\prime}$ de Latitud Sur y los meridianos $57^{\circ} 10^{\prime}$ y $58^{\circ} 40^{\prime}$ de Longitud Oeste.

Al norte limita con el Departamento Central, al Oeste con la República Argentina, separado por el río Paraguay, al Este con los Departamentos de Paraguari y Misiones y al Sur con la República Argentina separado por el río Paraná. (Gamarra Z. L., 2004)

Según Ramón Fogel. 2000, citado por Luisa Gamarra en Migraciones Interna Femenina:

El Departamento de Ñeembucú se encuentra constituido por dieciséis Distritos: Pilar, Alberdi, Cerrito, Laureles, Mayor José D. Martínez, Paso de Patria, San Juan Bautista, Humaitá, Villa Oliva, Isla Umbú, Villalbín, Desmochados, Gral. José E. Díaz, Tacuaras, Guazú Cúa, Villa Franca.

El clima es subtropical, donde la media anual es de $22,6^{\circ} \mathrm{C}$, mientras que las medias anuales varían entre $28,8^{\circ} \mathrm{C}$ en el mes de enero y $17,7^{\circ} \mathrm{C}$ en el mes de julio. La frecuencia de heladas va en aumento gradualmente hacia el sudoeste, registrándose una frecuencia media de dos veces al año.

La zona comprende una planicie baja aluvial caracterizada por sus inundaciones periódicas que provienen de los ríos Paraná y Paraguay. La cota oscila entre los 50 y 70 metros sobre el nivel del mar. La pendiente del lugar es de 1/10000 aproximadamente, y se presenta de Este a Oeste. En la margen derecha del río Paraná se observa una elevación de la topografía alcanzando a 75 metros sobre el nivel del mar como consecuencia de la acumulación de materia de sedimentación que se ha originado por la erosión de los suelos. El área de estudio está comprendida por una extensa planicie que cuenta, además del río Paraná y Paraguay, con esteros, arroyos con vegetación herbácea, de arbustos y bosques 
en galerías. Estos cursos de agua sirven para drenar las lluvias caídas, pero debido a la topografía del área, una gran parte del agua queda depositada en los esteros, pantanos y hondonadas. Esta región ha sido definida como sabana arbolada o formación termo hídrica a mesófila. (Fogel, 2000)

Los suelos predominantes son aluviales o transportados y se clasifican en grupos de alfisoles, entisoles y tierras misceláneas. En el orden de los alfisoles son suelos que dentro de los $75 \mathrm{~cm}$. de profundidad se presentan moteados de baja intensidad de color. Presentan condiciones ácuicas en los primeros horizontes dentro de los $75 \mathrm{~cm}$. La textura es francoarenosa. El contenido de materia orgánicas es de 1,2\% a menos de $20 \mathrm{~cm}$ de profundidad, el pH es ácido, bases de intercambio menor a 2 m.e. /100 gr. de suelo. Se encuentran localizados en la cuenca del arroyo Tebicuary; y en Pilar en el arroyo Yakaré y Mburicá. (Ibid)

\section{Importancia de la Apicultura}

Los productos de la abeja melífera han sido alimentos del hombre desde tiempos inmemorables, y siguen siéndolo en esta era moderna en que, con los adelantos tecnológicos, se ha logrado modernizar la explotación a un nivel tal que permite la obtención de grandes cantidades de los mismos; así como varios subproductos aplicables tanto a la alimentación humana y elaboración de medicamentos como en forma de ingredientes para muchas industrias como las de cosméticos y otras. (Arévalos, 2002)

De ahí su importancia desde el punto de vista económico, dado que constituye una fuente de ingreso interesante para el productor rural y los que participan en las diferentes etapas de los procesos de industrialización y de comercialización.

Pero quizás el mayor aporte de las abejas sea el de la polinización de las plantas, aunque puede ser el menos valorado por muchos. Mediante esta acción polinizadora de las abejas se obtienen mayores y mejores producciones, tanto de granos como de frutas. (Ibíd.)

La actividad apícola se encuentra desde hace algunos años en franca expansión. El notable crecimiento de la producción y exportación se debe al aumento de la demanda externa en los mercados tradicionales y en los nuevos. Este fenómeno se ve acompañado por la disminución de la cosecha de miel en los países productores por razones climáticas y sanitarias.

Es posible incorporar valor agregado a la actividad a través de distintos mecanismos de diferenciación. Estos pueden ser el fraccionamiento, la tipificación por origen botánico, 
la certificación de calidad por protocolos, la producción orgánica y el desarrollo de otros productos de la colmena como el polen, propóleos, jalea real, etc. Estos últimos cuentan con un importante potencial, ya que la demanda internacional es creciente e insatisfecha. (Gamarra K. , 2008)

\section{La Apicultura en el Paraguay}

Fueron los colonos europeos, sobre todo los alemanes, los que comenzaron a explotar en el país esta actividad, con muy buenos resultados.

Desde los años 1921 hasta el año 1928, el país llegó a ser exportador de miel de abeja al mercado europeo. Posteriormente, la Apicultura nacional experimentó un pronunciado bajón, debido principalmente a factores como:

- Falta de difusión de las técnicas de manejo de colmenas

- Dificultad para conseguir materiales e implementos básicos.

- Daños ocasionados por el empleo de insecticidas en los cultivos agrícolas.

- Falta de un Centro de Investigación y difusión de la cría de las abejas a nivel oficial.

- Carencia de especialistas que pudieran llevar adelante un Programa de Desarrollo Apícola.

- Invasión de las abejas africanizadas a partir del año 1965, aproximadamente.

- Falta de organización de los apicultores.

La recuperación de esta actividad se inició en el año 1970, mediante una acción conjunta del Ministerio de Agricultura y Ganadería, la Misión Técnica Japonesa, una agrupación de caracterizados apicultores nacionales y con la venida de experto Japonés. Así fueron creados los organismos que tuvieron una decidida participación en el desarrollo de esta actividad, como:

- El 10 de octubre del año 1971 fue fundada la Asociación de Apicultores del Paraguay.

- El 9 de julio del año 1973 fue creado el Departamento de Apicultura del Ministerio de Agricultura y Ganadería, según Decreto No 33.935

- Otra dependencia creada fue el Laboratorio de Apicultura de la Facultad de Ingeniería Agronómica que, además de realizar la cría y distribución de reinas seleccionadas a los productores, realiza trabajos de investigaciones, análisis de productos y enseñanza de técnicas apícolas. 
Además, cabe destacar la valiosa cooperación de la Misión técnica Japonesa que fue de 10 años, en su primera etapa, desde el año 1970 hasta el año 1980. Esta cooperación se ha reiniciado en su segunda etapa en el año 1988.

También es importante señalar la cooperación recibida de parte del gobierno del Japón, a través de la Agencia de Cooperación Internacional del Japón (JICA), con el envío de Expertos en Apicultura, equipamiento del Laboratorio de Apicultura de la Facultad, cursos de especialización para profesionales paraguayos y así también la valiosa colaboración de parte de la Asociación Suiza para el Desarrollo y la Cooperación (HELVETAS)

En los últimos 15 años, el número de colmenas de abejas registradas tuvo un incremento del $257 \%$ y el número de apicultores, es de 623\%. (Apicultura Moderna, 2da Ed.)

\section{Legislación apícola en el Paraguay}

Por Decreto No 33.935, del 9 de julio del año 1973, se ha creado el Departamento de Apicultura, como una dependencia técnica del Ministerio de Agricultura y Ganadería. Esta dependencia tiene como objetivo: orientar e incentivar la actividad apícola del país. Además de prestar atención a los problemas apícolas nacionales y tomar las medidas necesarias en defensa de los productores.-

Posteriormente, el Congreso Nacional sancionó la Ley Nº66, el 9 de Diciembre de 1977, la cual rige actualmente. Esta Ley declara a la apicultura como una actividad de importancia económica y social, como así también la protección de la abeja melífera como insecto útil.

La creación del departamento de apicultura y la promulgación de la ley $\mathrm{N}^{\mathrm{o}} 665$ son factores de importancia para el quehacer apícola nacional, en donde están sentadas las bases para su desarrollo, ya que los productores son los beneficiados directos.(Ibíd.)

\section{ESTRATEGIAS METODOLÓGICAS O MATERIALES Y MÉTODOS}

Para el estudio del trabajo, se toma las zonas de mayor producción y volumen que se encuentra localizadas en las zonas de General J. E. Díaz, Paso de Patria y Humaitá. Estas son ciudades ubicadas en el sur del Departamento del Ñeembucú. 


\section{Gráfico 1}

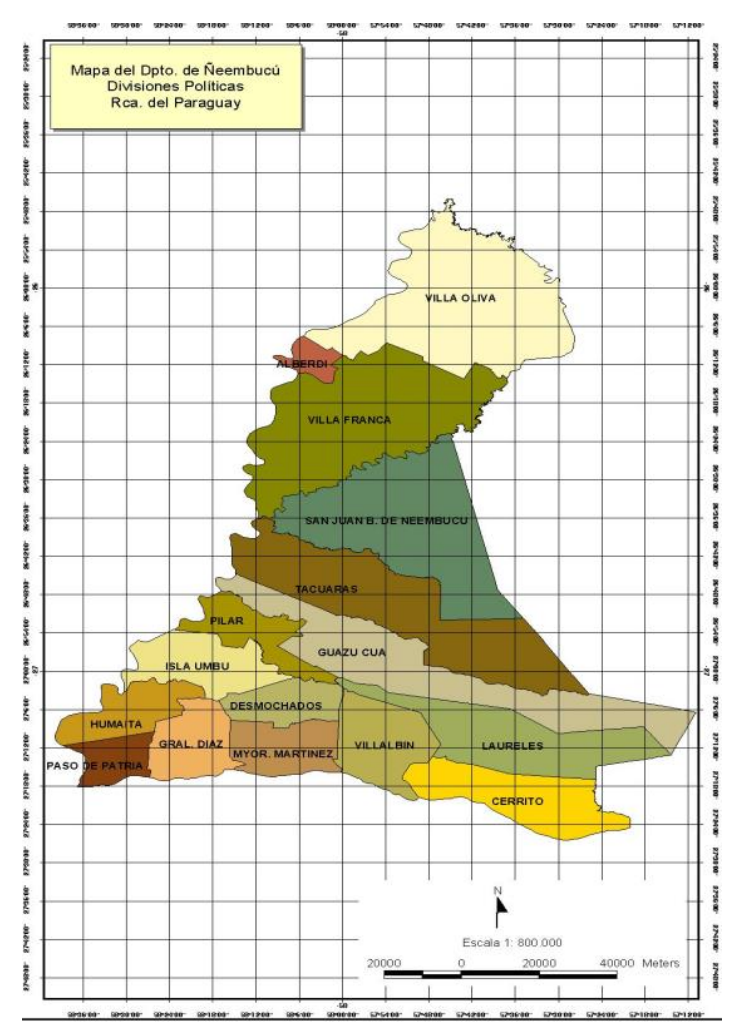

El trabajo se encuadró en la tipología de estudio: Descriptiva

El estudio denominado "descriptivo" estuvo dirigido a caracterizar la producción apícola e identificar factores asociados a la situación que enfrenta la producción y por ende su calidad.-

Para los fines que interesó al presente trabajo, si bien el departamento del Ñeembucú cuenta con mas de 250 apicultores, el universo o población tomado como base para el estudio, estuvo conformado por las zonas de mayor producción Gral. Díaz, Paso de Patria, Humaitá. Para llevar adelante esta investigación se implementó como técnica de recolección de datos la entrevista a productores, técnico apicultor y finalmente el análisis laboratorial para caracterizar la calidad de la miel.

\section{RESULTADOS Y DISCUSIÓN}

Se concluye el trabajo, de conformidad a los resultados alcanzados, teniendo en cuenta los objetivos, el problema de investigación. Finalmente se realiza sugerencias sobre la base de las falencias detectadas de conformidad a los hallazgos de la investigación.

El cultivo de miel se encuentra localizado en los distritos de Isla Umbù, Humaità, Paso de Patria, Gral. Díaz, Desmochados, Mayor Martínez, Villabìn, Cerrito y Laureles, destacándose entre ellos Gral. Díaz, Humaitá y Paso de Patria como las zonas de mayor 
Producción y volumen. (DEAG, Lic. Atilio Benítez, técnico del DEAG, comunicación personal). Es necesario destacar que estas zonas están ubicadas en zonas de carrizales y los Ríos Paraná y Paraguay.

En cuanto al volumen de producción actual, la misma ha sufrido una disminución con relación al periodo de producción de la década del año 90 y 200, a causa de los fenómenos climáticos de mucha precipitación en la primera etapa de producción y una sequía prolongada en la etapa final, que fue determinante en la merma de producción, además de los ataques de enfermedades como la Loque Europea y polillas, que son plagas que ocasionaron grandes pérdidas económicas y que redujo en un $50 \%$ del total de 30 ton producidas por año. (Benítez, 2017)

En cuanto al sistema de comercialización implementada por los productores es de carácter informal con acopiadores informales y con empresas envasadoras de la capital en granel o por $\mathrm{Kg}$. Así también la venta se realiza de forma individual en negocios de consumo de Pilar y a través de ferias agropecuarias.

\section{Gráfico 2}

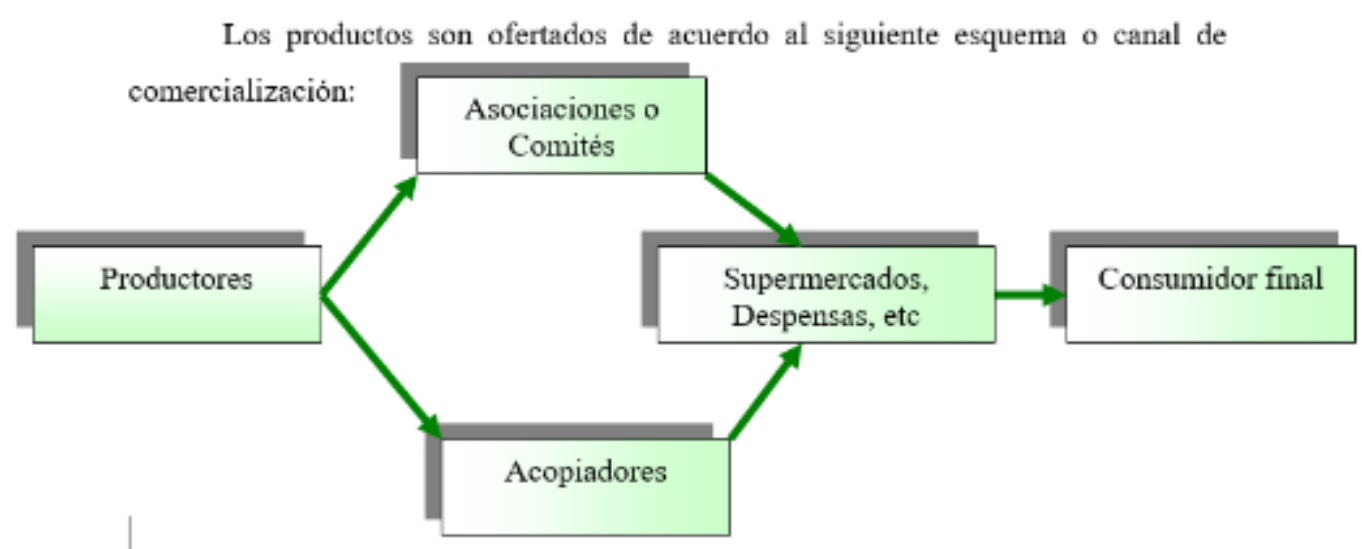

Fuente: Elaboración propia

Durante la entrevista realizada a especialistas apicultores, se pudo conocer que los implementos y materiales utilizados por los productores son básicos

Respecto a la calidad de la miel teniendo en cuenta las características de la vegetación en las tres localidades, se destaca que la miel obtenida en el Departamento es considerada por los apicultores y empresas comercializadoras $100 \%$ puro, de excelente calidad por las características mismas de la zona de cultivo. En las tres localidades ( zonas de estudio) no existe diferencia alguna en su vegetación, poseen la misma plantación como ser el Césamo, lapacho, Ingá, entre otros. 
De acuerdo a los resultados laboratoriales, se tuvo en cuenta los datos proveídos por el Centro de Desarrollo Agropecuario- Neembucú.

Tabla 1

\begin{tabular}{|l|c|c|c|}
\hline \multicolumn{1}{|c|}{ Muestras } & Humedad \% & $\begin{array}{c}\text { Azucares } \\
\text { reductores \% }\end{array}$ & $\begin{array}{c}\text { Sólidos insolubles } \\
\text { en agua \% }\end{array}$ \\
\hline Humaitá & 19,2 & 75,0 & 0,08 \\
\hline General Díaz & 19,6 & 73,5 & 0,09 \\
\hline Paso de Patria & 19,7 & 74,3 & 0,1 \\
\hline Norma de Calidad & Máx 21\% & Mín 65\% & Máx 0,1\% \\
\hline
\end{tabular}

La humedad en la miel es utilizada como un indicador de la madurez y capacidad de permanecer estable durante el almacenamiento. Se ha reportado que mieles obtenidas durante periodos de altas precipitaciones (época de lluvias) presentan un mayor contenido de humedad que las mieles producidas durante épocas de bajas precipitaciones (sequía)

\section{CONCLUSIÓN O CONSIDERACIONES FINALES}

Las tendencias actuales de los mercados exigen la producción de alimentos inocuos y genuinos. Si la calidad de un producto se relaciona con el cumplimiento de las características esperadas por los consumidores y la incorporación de las nuevas y cambiantes exigencias, para el logro de la misma deben considerarse las acciones tomadas desde la obtención de la materia prima hasta la venta del producto final. Estos cuidados pueden contribuir a abrir nuevos mercados donde la miel sea reconocida por sus características diferenciales.

Una forma efectiva de lograr la satisfacción del cliente/ consumidor es mediante la aplicación de sistemas de aseguramiento de la calidad, herramientas indispensables a la hora de comercializar un alimento.

La falta de un Centro de Acopio y envasado de miel en el Departamento hace que el producto sea trasladado a granel sin un valor agregado por las empresas capitalinas o en otros departamentos, países vecinos, quedando la mayor parte del beneficio económico fuera de nuestra región.

Para identificar las características de la producción de miel, se recurrió a bibliografías y se tuvo en cuenta las Normativas reguladas por la Comisión Codex Alimentarius- Normas Internacionales de Alimentos, y establecido en el código Codex Stan 12-1981. En base a los resultados de los diferentes instrumentos de recolección de datos se puede determinar 
que la miel de abeja producida en el departamento de Neembucú, cumplen con las normas de calidad establecidas.

En cuanto a la caracterización de valores de humedad, fueron extraídas mieles de los distritos estudiados y sometidos a prueba de análisis laboratoriales, que demuestran que el porcentaje de humedad de la miel de abeja, se encuentra dentro de las normativas establecidas, por lo que el producto es considerado bueno. En cuanto a la cantidad de azúcares, tienen características normales establecidas por la Codex Stan 12-1981. Esto de acuerdo a la entrevista a los apicultores, se debe fundamentalmente por el tipo de vegetación que abunda en la zona. Así mismo, la cantidad de sólidos insoluble en agua, se adecuan a las normas de calidad establecida.

\section{LISTA DE REFERENCIAS}

Abril., M. V. (2014). Estudio e Identificación de caracteristicas de composición y bioactividad propias de miel mielato Apis Mellifera. Universidad Nacional de Colombia, Bogotá, Colombia

Arevalos, Porfirio. Estudio de suelo. Características físico - químicas de los suelos del área del Proyecto DERMASUR. Porfirio Arévalos y Shizuo Sunaga. MAG JICA. Pilar, Paraguay. 1999

Arevalos, Porfirio. Evaluación de la Tecnología Agrícola utilizada por pequeños productores. El rubro Algodón. UNP. EPG. Pilar. Paraguay. 2002

Bernal, César. Metodología de la Investigación: para administración y economía. César Augusto Bernal T. PEARSON. Bogotá. 2000

Benítez, L. (2017). Calidad de la miel producida en el departamento de Ñeembucú. Pilar.

Camaño, C. A. (2003). Determinacion del origen floral y caracterizacion fisico y quimica de las mieles (Alpis Mellifera). Obtenido de http://www.pfnm.cl/paqtecnologicos/ulmo/caracterizacion-miel.pdf.

Fogel, R. (2000). La Ecorregión del Neembucú: Infortunio, dignidad y sabiduría de sus antiguos pobladores. Asunción.

Formicheli, Paulo. (2002)Procesos de diferenciación de pequeños productores campesinos de la región sur de Pilar. UNP. EPG. Pilar - Paraguay. 2002

Gamarra, K. (2008). Desarrollo Apícola en el Departamento de Neembucú. Pilar: UNP.

Gamarra, Z. L. (2004). Migración Interna femenina en el departamento de Ñeembucú. Causas y Efectos. Pilar. 
Galeano de Ferreiro, Eliodora. La Villa del Pilar que nunca vuelve. Pilar.1994

Gómez D., José María. (2001) Metodología de la Investigación. Apuntes para el Aula. Pilar - Paraguay. 99 p.

Miranda. E (2005). Metodología de la investigación cuantitativa y cualitativa.

Moguel Ordóńeza Y, Echazarreta Gonzalez C, Mora Escobedo R. (2005). Calidad fisicoquímica de la miel de abeja Apis mellifera producida en el estado de Yucatán durante diferentes etapas del proceso de producción y tipos de floración. Técnica Pecuaria en México, 43(3), 323-324.

MAG -JICA. Estudio Socio- Económico de productores del área del Proyecto DERMASUR. Pilar, 1999

Tamayo y Tamayo. El proceso de la Investigación científica. LIMUSA. 3ra ed. México. 199

Quiñónez, Mario Ricardo. Apicultura Moderna. 2a Ed. San Lorenzo - Paraguay. 1993

Sampieri Hernández, R (1998). Metodología de la Investigación. Editorial McGraw- Hill. S.A

Sans, S. S., \& Sans, M. M. (1994). Humedad, cenizas y conductividad eléctrica de mieles de La Rioja. Dialnet, 143-158.

Simal, J. y Huidobro, J.F (1984). Parámetros de calidad de la miel III: acidez (pH, libre, lactónica y total) e índice de formol. 